\begin{tabular}{|c|l|}
\hline Title & Surface Temperature Dependence of Hydrogen Ortho-Para Conversion on A morphous Solid W ater \\
\hline Author(s) & Ueta, Hirokazu; W atanabe, Naoki; Hama, Tetsuy a; Kouchi, A kira \\
\hline Citation & $\begin{array}{l}\text { Physical Review Letters, 116(25), 253201 } \\
\text { https://doi.org/10.1103/PhysRevLett.116.253201 }\end{array}$ \\
\hline Issue Date & 2016-06-23 \\
\hline Doc URL & http://hdl.handle.net/2115/62697 \\
\hline Rights & @2016 A merican Physical Society \\
\hline Type & article \\
\hline Additional Information & There are other files related to this item in HUSCAP. Check the above URL. \\
\hline Information & PhysRevLett.116.253201(1).pdf \\
\hline
\end{tabular}

Instructions for use 


\title{
Surface Temperature Dependence of Hydrogen Ortho-Para Conversion on Amorphous Solid Water
}

\author{
Hirokazu Ueta ${ }^{\dagger}$ Naoki Watanabe, ${ }^{*}$ Tetsuya Hama, and Akira Kouchi \\ Institute of Low Temperature Science (ILTS), Hokkaido University, Sapporo 060-0819, Japan
}

(Received 26 November 2015; published 22 June 2016)

\begin{abstract}
The surface temperature dependence of the ortho-to-para conversion of $\mathrm{H}_{2}$ on amorphous solid water is first reported. A combination of photostimulated desorption and resonance-enhanced multiphoton ionization techniques allowed us to sensitively probe the conversion on the surface of amorphous solid water at temperatures of 9.2-16 K. Within a narrow temperature window of $8 \mathrm{~K}$, the conversion time steeply varied from $\sim 4.1 \times 10^{3}$ to $\sim 6.4 \times 10^{2} \mathrm{~s}$. The observed temperature dependence is discussed in the context of previously suggested models and the energy dissipation process. The two-phonon process most likely dominates the conversion rate at low temperatures.
\end{abstract}

DOI: 10.1103/PhysRevLett.116.253201

The hydrogen molecule holds a fascination for scientists in physics and chemistry, but also in astronomy, because of its quantum mechanical nature and massive abundance in space. The rotational states of molecular hydrogen couple with nuclear spins. Ortho- and parahydrogen with parallel and antiparallel proton spins have odd and even rotational states, respectively. Because a rotational energy difference of $\sim 14.7 \mathrm{meV}$ corresponds to a temperature of $170 \mathrm{~K}$ between the lowest para $(J=0)$ and ortho $(J=1)$ states, which is significantly higher than the local temperature $(\sim 10 \mathrm{~K})$ in interstellar molecular clouds, ortho- and parahydrogen play different roles in the chemical reactions occurring in those environments [1]. The radiative ortho-topara (OP) conversion in the gas phase is forbidden, and thus extremely slow. Therefore, it is highly desirable to know how the OP conversion occurs on the surfaces of cosmic dust solids where $\mathrm{H}_{2}$ is produced.

In contrast to the gas phase, OP conversion is promoted on various solid surfaces [2,3]. On magnetic surfaces and/or with magnetic impurities, such as $\mathrm{O}_{2}$, the $\mathrm{OP}$ conversion is induced via magnetic dipole interactions $[4,5]$. Furthermore, conversion on diamagnetic surfaces such as Ag [4-9], $\mathrm{Cu}[10,11]$, and graphite $[12,13]$ have been reported. The proposed conversion mechanism for those surfaces is the Coulomb contact model [3]. Recently, experimental studies demonstrated that dielectric and diamagnetic insulators of the microporous material MOF-74 [14] and amorphous solid water (ASW) [15-18] can also induce the OP conversion of $\mathrm{H}_{2}$. This $\mathrm{H}_{2}-\mathrm{ASW}$ system is of great astronomical interest because hydrogen molecules are considered to form on cosmic dust covered by ASW in interstellar molecular clouds [19]. Therefore, understanding OP conversion on ASW would greatly impact the study of chemical evolution in interstellar clouds.

As a possible mechanism of OP conversion on ASW, Sugimoto and Fukutani [17] proposed that the presence of giant inhomogeneous surface electric fields with steep gradients on the surface of ice [20] can induce a change of rotational states via the Stark effect, and thus enhance spin-orbit couplings between electronic states of molecules. By these effects, nuclear spins can change via intramolecular hyperfine contact interactions. Although an excellent conversion mechanism has been proposed, other surface properties and processes should play a role in the conversion. For example, it is known that the surface of ASW has a broad range of adsorption sites [21]. The magnitude of the surface electric field and its gradient may depend on the site where $\mathrm{H}_{2}$ adsorbs. Thus, the OP conversion rate may depend on the adsorption site. Furthermore, in the OP conversion, excess energy that is equivalent to the energy difference of two states must be released. While this process has not been paid much attention for the OP conversion at surface, previous studies showed the importance of energy dissipation for the OP conversion in matrices at low temperatures [22-25]. To obtain deeper insight into the entire conversion mechanism, we investigated the surface-temperature dependence of OP conversion on ASW in the time scales from 10-600 s. We found that the conversion rate varies steeply with surface temperature in the narrow range of 9.2-16 K. The obtained temperature dependence indicates that the conversion rates at low temperatures are dominated by two-phonon energy dissipation process.

The experiments were performed in an ultrahigh vacuum chamber at a base pressure of $\sim 3.0 \times 10^{-8} \mathrm{~Pa}$ with a doubly differentially pumped molecular beam source. The ASW films were grown to a thickness of approximately 20 monolayers on an aluminum substrate at a surface temperature $\left(T_{\mathrm{S}}\right)$ of $10 \mathrm{~K}$ by introducing carefully degassed $\mathrm{H}_{2} \mathrm{O}$ vapor into the vacuum chamber through a leak valve $\left(2.0 \times 10^{-5} \mathrm{~Pa} \times 300 \mathrm{~s}\right)$. The surface area of the ASW with this thickness is about 10 times larger than a well-packed flat surface that has typical adsorption sites of $10^{15} \mathrm{~cm}^{-2}$ [26]. Temperature was measured with a Si diode 
and controlled with fluctuation of less than $\pm 0.5 \mathrm{~K}$. Before each experiment, the sample was heated to $55 \mathrm{~K}$ to eliminate any existing $\mathrm{O}_{2}$ molecules from the ASW surface $[17,27]$, as these are known to promote nuclear spin flip on the surface [15]. The pulsed molecular beam of normal pure $\mathrm{H}_{2}$ with an OP ratio of 3 struck the ASW surface with a beam spot of $3 \mathrm{~mm} \phi$. An incident angle is $45^{\circ}$ with respect to the surface normal. This $\mathrm{H}_{2}$ deposition method has the great advantage of shortened deposition times, and avoids any increase of undesired background $\mathrm{H}_{2}$ in the chamber. If $\mathrm{H}_{2}$ is deposited by a gas flow through a nozzle, the $\mathrm{H}_{2}$ gas spreads widely beyond the sample area and a significant amount of the gas eventually permeates the chamber. Such an $\mathrm{H}_{2}$ gas can immediately undergo OP conversion on the metal surfaces of cold head and shroud [11] and return to the sample surface even during the deposition, which may poison the initial ortho-para ratio of the samples. Adsorbed ortho- and parahydrogen were detected by a combination of photostimulated desorption (PSD) and $(2+1)$ resonanceenhanced multiphoton ionization (REMPI) techniques via the $E, F^{1} \sum_{g}^{+}$states with a time-of-flight mass spectrometer $[16,18]$. The PSD was caused by irradiation of a nonfocused pulsed laser light at a wavelength of $532 \mathrm{~nm}$ and energy of about $163 \mu \mathrm{J}$ pulse $^{-1}$. We confirmed that the PSD laser irradiation did not cause undesired chemical process effects and structural changes of the ASW [16]. The REMPI-laser irradiation fixed at resonance wavelengths for the ionization of $\mathrm{H}_{2}(v=0 ; J=0$ or 1$)$ were focused at a distance of about 5-6 $\mathrm{mm}$ above the sample surface. Both PSD and REMPI lasers were run at a repetition rate of $10 \mathrm{~Hz}$, and the delay time between both laser pulses was fixed to a time when the detected desorbed hydrogen signal was at a maximum. In these experiments, $\mathrm{H}_{2}$ molecules in the $J=2$ and 3 states were not detected. The total impinging doses of $\mathrm{H}_{2}$ were of the order $10^{15}$ molecules $\mathrm{cm}^{-2}$, with deposition times of 2.5-4 s. The $\mathrm{H}_{2}$ surface density decreased with an increase of surface temperature due to different sticking coefficients. The relative $\mathrm{H}_{2}$ densities were estimated from the total desorption signals, to vary from 1 to 0.3 at $10-16 \mathrm{~K}$, respectively. At temperatures above $16 \mathrm{~K}$, the $\mathrm{H}_{2}$ surface density drops rapidly because of molecular desorption and thus the measurement was not possible.

The measurements were performed in temperature range from 9.2 to $16 \mathrm{~K}$. With starting the $\mathrm{H}_{2}$ deposition, the REMPI laser was continuously injected above the ice surface so that $\mathrm{H}_{2}$ signal is immediately recorded once $\mathrm{H}_{2}$ photodesorbs from the ice. After certain periods of residence time, $\mathrm{H}_{2}$ was photodesorbed and, consequently, ionized state selectively at either $J=0$ or 1 by REMPI. The PSD laser injection was continued for $60 \mathrm{sec}$ until photodesorbed $\mathrm{H}_{2}$ intensity decreased significantly (see Supplemental Material [28]). Figure 1 shows a series of $J=0$ and $J=1$ REMPI signals for residence times of 10,300 , and $600 \mathrm{~s}$ after the $\mathrm{H}_{2}$ deposition at $T_{S}=16 \mathrm{~K}$. Area intensities for REMPI signals

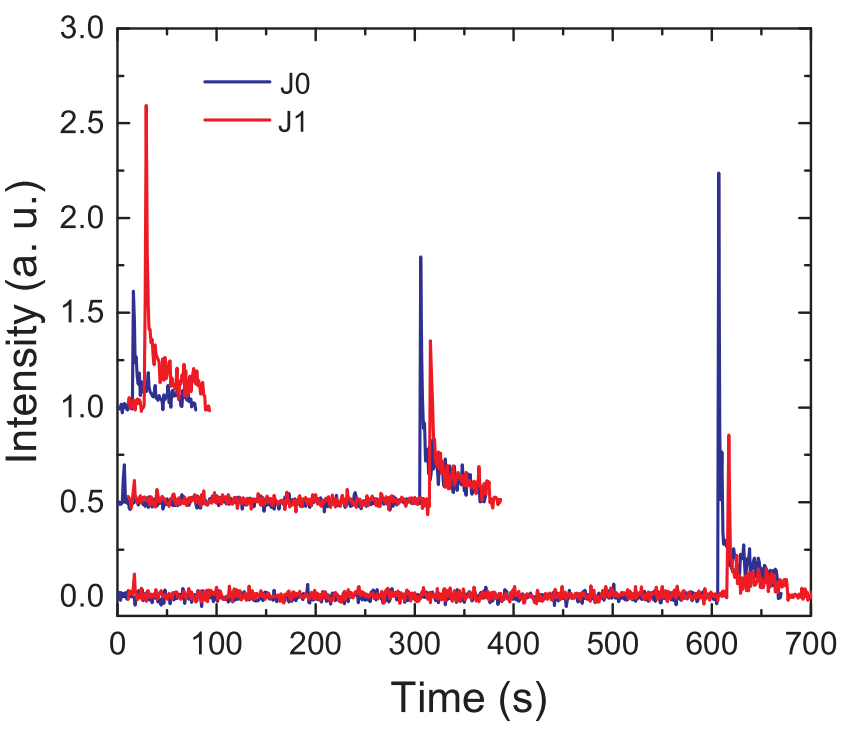

FIG. 1. Intensities of photodesorbed $\mathrm{H}_{2}$ for $J=0$ (blue) and 1 (red) detected by REMPI at different residence times (from top 10,300 , and $600 \mathrm{~s}$ ). The $\mathrm{H}_{2}$ was deposited on the ASW at $16 \mathrm{~K}$. The time scale of the $x$ axis includes a deposition time of $2.5 \mathrm{~s}$. For clarity, the data sets for the time of 10 and $300 \mathrm{~s}$ are offset by 1.0 and 0.5 at the $y$ axis, respectively. The time axis of the $J=1$ data have been shifted by $10 \mathrm{~s}$. The REMPI laser was injected above the ice surface even during the residence time but the signal of $\mathrm{H}_{2}$ was not detected (base lines) until the injection of the PSD laser.

in Fig. 1 are plotted in Fig. 2. As shown in Fig. 2, the sum of the $J=0$ and 1 signals are constant within the experimental duration, so that the change in the $J=0$ and 1 signal intensities directly corresponded to the OP conversion rate at the surface. At $t=10 \mathrm{~s}$, the $J=1$ signal intensity was higher than that measured for $J=0$. However, at $t=600 \mathrm{~s}$ the intensity of the $J=0$ signal dramatically increased over that of $J=1$, indicating the occurrence of the OP conversion. The changes of signal intensities are plotted in Fig. 2 as a function of residence time at different $T_{S}$. The time in the plots includes the beam deposition time up to $4.0 \mathrm{~s}$. At all $T_{S}$ values, the intensity of the $J=1$ signal decreased with increasing time, while the $J=0$ signal increased over the same time period. The OP conversion rate is obviously different at each surface temperature. The conversion time can be determined by fitting the data for $J=1$ with a single exponential function. The conversion time constants are determined to be $4.1 \pm 1.9 \times 10^{3}, 3.1 \pm$ $0.7 \times 10^{3}, 1.6 \pm 0.3 \times 10^{3}, 1.4 \pm 0.1 \times 10^{3}, 7.5 \pm 0.8 \times 10^{2}$, $5.8 \pm 0.3 \times 10^{2}$, and $6.4 \pm 0.4 \times 10^{2}$ s for $9.2,10,10.5,11$, 12,14 , and $16 \mathrm{~K}$, respectively and plotted in rates $\left(\mathrm{s}^{-1}\right)$ as a function of $T_{S}$ in Fig. 3. The conversion rate steeply increased with increasing $T_{S}$ even within the narrow temperature window used in the experiment. For comparison purposes, the nuclear spin conversion was also measured for $\mathrm{D}_{2}$ molecule at $T_{S}=16 \mathrm{~K}$ (Supplemental Material [28]). The conversion was obviously much slower than that 

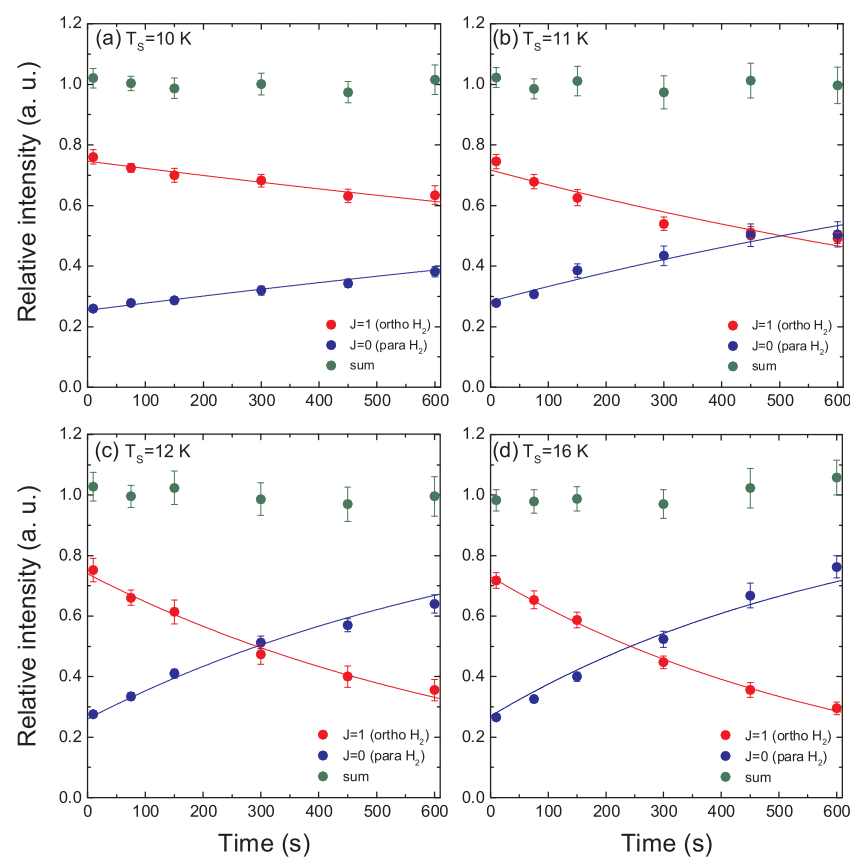

FIG. 2. The time evolution of the photodesorbed $\mathrm{H}_{2}$ intensities for $J=0$ (blue), 1 (red), and their sum (green). Intensities were derived from the integrated photodesorbed $\mathrm{H}_{2}$ signals. The surface-temperature dependence of $\mathrm{H}_{2}$ is shown for (a) 10, (b) 11 , (c) 12 , and (d) $16 \mathrm{~K}$. The origin of the $x$ axis corresponds to the opening of the pulsed valve of the molecular beam source. The error bars represent the confidence reliabilities for 6 to 9 measurements. Solid lines are results of single-exponential fittings.

for $\mathrm{H}_{2}$ at $T_{S}=16 \mathrm{~K}$ which is also consistent with previous experiments [15-17].

One scenario that may explain the observed strong temperature dependence of the conversion rate is that the conversion is sensitive to adsorption potential sites on the surface. Sugimoto and Fukutani proposed that the conversion is triggered by inhomogeneous giant electric fields on the ASW surface [17]. More recently, it was reported that the OP conversion efficiency of $\mathrm{H}_{2}$ in a Hofmann-type porous coordination polymer depends on $\mathrm{H}_{2}$-trapped sites in bulk [29]. The efficiency was found to be enhanced at the site where the magnitude of the local electric field is stronger. In their experiment, the distribution of $\mathrm{H}_{2}$ molecules at each trapped site can be controlled via the bulk temperature. Owing to the amorphous structure, adsorption sites have various potential depths on the ASW surface [21] where the magnitude of the electric field should differ with each other. The $\mathrm{H}_{2}$ molecules diffuse rapidly on ASW and are finally trapped at adsorption sites with a certain distribution depending on the surface temperature $[21,30]$. Therefore, the conversion rate can depend on the adsorption site(s) on ASW. The deep adsorption potential site is considered to originate from defects, dips, and steps on the surface of ASW. Therefore, $\mathrm{H}_{2}$ molecules in such deep sites interact strongly with the surrounding $\mathrm{H}_{2} \mathrm{O}$ via dangling $\mathrm{OH}$ and thus suffer from electric fields with larger magnitudes, which would induce a faster OP conversion. Since the $\mathrm{H}_{2}$-desorption temperature from ASW is around $20 \mathrm{~K}$, at temperatures close to $20 \mathrm{~K}$ the $\mathrm{H}_{2}$ molecules cannot stay in shallower sites and thus tend to be located at the deep adsorption potential sites. In contrast, the $\mathrm{H}_{2}$ molecules can be trapped even at shallower sites at $10 \mathrm{~K}$, which is well below the desorption temperature. This temperaturecontrolled $\mathrm{H}_{2}$ adsorption site may make the conversion rate faster at higher temperatures. To confirm this scenario, we first deposited $\mathrm{H}_{2}$ molecules on ASW at 12 and $16 \mathrm{~K}$ where $\mathrm{H}_{2}$ stays only at deeper sites. After the deposition, the surface temperature quickly decreased to $10 \mathrm{~K}$, where $\mathrm{H}_{2}$ trapped in deep sites at the deposition cannot move. In this manner, the $\mathrm{H}_{2}$ distribution on the surface sites produced at 12 and $16 \mathrm{~K}$ can be frozen at $10 \mathrm{~K}$. If deep-potential sites play an important role in the enhancement of the conversion rate above $12 \mathrm{~K}$, a fast conversion should appear on the $10-\mathrm{K}$ ASW. However, the OP conversion rate obtained from this measurement was almost equivalent to that for the $10 \mathrm{~K}$ deposition case shown in Fig. 2(a); see the Supplemental Material [28]. This result indicates that the conversion rate cannot be explained solely by the difference in the potential sites, and instead an additional mechanism is required.

The OP conversion of molecules on the surface is completed by the transformation of nuclear spin states, in other words the nuclear spin flip and the subsequent energy dissipation. Therefore, the role of phonons in the energy dissipation process should be considered when explaining the temperature dependence of the conversion. For $\mathrm{H}_{2}$ physisorbed on ASW, the energy difference between the $J=1$ (ortho) and 0 (para) states, which would be rather smaller than that of $14.7 \mathrm{meV}$ for isolated due to the suppression of rotational motion on the ASW, must be released through the surface and/or bulk phonon processes. Spin-lattice relaxation occurs via one-phonon or twophonon processes. The rate $f_{\text {one }}$ of the one-phonon process is proportional to

$$
f_{\text {one }} \propto \operatorname{coth}\left(\frac{\delta}{2 k T}\right),
$$

where $\delta$ is the energy difference between two spin states, and $T$ is the solid temperature [24]. In the present experiment, taking $\delta=14.7 \mathrm{meV}$, which corresponds to $170 \mathrm{~K}$ and $T=T_{S} \leq 16 \mathrm{~K}$, the rate of the one-phonon process becomes almost temperature independent, which cannot explain the temperature dependence observed here. The two-phonon process proceeds via the simultaneous absorption of a phonon from the initial up to intermediate states, and the emission of another from an intermediate to the final states, which can be categorized into Orbach or Raman process when the intermediate state is a real or virtual state, respectively [24]. These two-phonon relaxation mechanisms were found to be applicable for explaining 


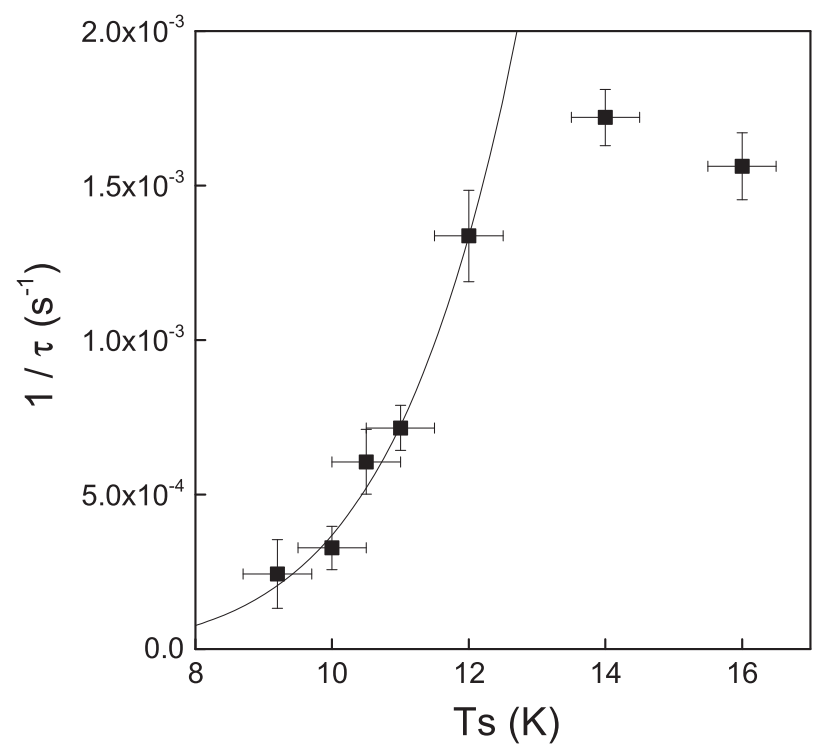

FIG. 3. Temperature dependence of the OP conversion rate for $\mathrm{H}_{2}$ on ASW. Solid line represents the result of fitting the data with a power law of the form $A T^{n}$. Values of $A=3.2 \times 10^{-11} \mathrm{~s}^{-1}$ and $n=7.1 \pm 0.6$ provided the best fit.

the temperature dependence of nuclear-spin conversion, coupled with rotational states, of $\mathrm{CH}_{4}$ in solid parahydrogen [23], and also that of rovibrational dephasing of $\mathrm{CD}_{4}$ in a para- $\mathrm{H}_{2}$ matrix [22]. For the OP conversion of $\mathrm{H}_{2}$, the intermediate real state is $J=2$. Since the energy difference between $J=1$ and 2 is approximately $29.4 \mathrm{meV}(\sim 341 \mathrm{~K})$ in the gas phase, which is far above the Debye temperature of water ice [31], the Orbach process is not suitable in the present case. In contrast, the Raman process, which only requires the energy difference of two phonons to be equivalent to that between the initial and final states, can be considered as a plausible relaxation process. In fact, in an inelastic neutron-scattering experiment [32], acoustic phonon modes of vapor-deposited ASW were observed at $50 \mathrm{~K}$ to cover energies below approximately $23 \mathrm{meV}$ with two very broad peaks centered at around 7 and $17 \mathrm{meV}$. A phonon mode at $\sim 6 \mathrm{meV}$ was also observed in a surfacesensitive He-scattering experiment [33]. These phonons enable the OP conversion of $\mathrm{H}_{2}$ on ASW via the Raman process. Since the rate of the Raman process is proportional to $T^{n}$ at low temperatures $[24,34]$, we fitted the data of the temperature dependence with a power law of the form $A T^{n}$. The fitting failed when including the plots at 14 and $16 \mathrm{~K}$ but, taking $A=3.2 \pm 4.8 \times 10^{-11} \mathrm{~s}^{-1}$ and $n=7.1 \pm 0.6$ reproduces the experimental data below $14 \mathrm{~K}$ very well, as shown in Fig. 3. The power of $n=7$ is particularly known to appear in the Raman process when the temperature is sufficiently lower than the Debye temperature of the given solid [34], which suggests that the OP conversion rate is limited by the energy dissipation process at temperatures below $14 \mathrm{~K}$. The reason for deviating from the power law at above $14 \mathrm{~K}$ is unclear (Fig. 3), but it implies that the OP conversion rate above $14 \mathrm{~K}$ is limited by the nuclear spinflip rate by the ortho-para state mixing before the energy dissipation by phonons.

An alternative explanation for OP conversion was recently given by Ilisca and Ghiglieno [35]. According to their model, electrostatic and dynamical moleculesurface interactions play an important role in the conversion. Coulomb repulsion induces exchanges of $\mathrm{H}_{2}$ and surface electrons, leading to virtual excitation to triplet states, and the hyperfine contact interaction leads to nuclear spin flips. Furthermore, it was addressed that short-time interaction between hydrogen molecules and the repulsive part of the surface potential, such as (repetitive) surface scattering, accelerates the conversion. However, in the present experiment, the $\mathrm{OP}$ ratio at residence time of $\sim 10$ s was 3 for normal $\mathrm{H}_{2}$ molecules within the experimental accuracy. This implies that the initial scattering process, including single collisions, is not efficient to promote OP conversion of adsorbed $\mathrm{H}_{2}$ molecules.

Here, the astronomical implications of our result are described. The $\mathrm{OP}$ ratio of $\mathrm{H}_{2}$ is key for understanding chemical evolution in cold interstellar clouds because of the large energy difference $(\sim 170 \mathrm{~K})$ between the $J=0$ and 1 states, compared to the temperature of the environment. For example, the ion-molecule reaction,

$$
\mathrm{H}_{3}^{+}+\mathrm{HD}(J=0) \rightarrow \mathrm{H}_{2} \mathrm{D}^{+}+\mathrm{H}_{2},
$$

is very important to produce $\mathrm{H}_{2} \mathrm{D}^{+}$, which further produces other deuterated molecules via deuteron transfer. Reaction (2) is exothermic by about $230 \mathrm{~K}$, and works as an $\mathrm{H}_{2} \mathrm{D}^{+}$ formation route when the reactant $\mathrm{H}_{3}^{+}$and the products are in the para ground state. However, when the reactants, $\mathrm{H}_{2} \mathrm{D}^{+}$and $\mathrm{H}_{2}$, are both in the lowest ortho state, the reverse process of reaction (2) with para- $\mathrm{H}_{3}^{+}$production becomes efficient, even in $10 \mathrm{~K}$ clouds. That is, ortho $\mathrm{H}_{2}$ can be a chemical poison to molecular deuteration in clouds [1]. The present results first demonstrate that the OP ratio of $\mathrm{H}_{2}$ when released to the gas phase from cosmic dust strongly depends on the dust temperature and the duration at the surface. It should be noted that at certain temperature and morphology of dust surface the desorption rate of $\mathrm{H}_{2}$ formed on dust can be larger than the conversion rate.

In summary, we measured the temperature dependence of $\mathrm{H}_{2} \mathrm{OP}$ conversion on ASW. At temperatures below $14 \mathrm{~K}$, the conversion rate accelerates with surface temperature. This steep temperature dependence can be fitted as a power law that depends on $T_{S}$, which can be explained by the twophonon energy dissipation process. Above $14 \mathrm{~K}$, the conversion rate deviates from a power law, which may be limited by the rate of nuclear spin flip. Considerable progress has been made on the theoretical study of gassurface dynamics and surface scattering study in the last decade, in particular hydrogen with metal surfaces [36,37]. Such theoretical and experimental studies can help to better understand the interaction between hydrogen and ice. 
This work was partly supported by a Grant-in-Aid for Scientific Research from the Japan Society for the Promotion of Science (JSPS; Grant No. 24224012) and by a research fellowship from JSPS for Young Scientists (H. U.). We wish to extend our thanks to Dr. H. Hidaka, Dr. Y. Oba (ILTS), Dr. Y. Nakai (RIKEN), and Professor O. Yamamuro (ISSP) and the staff of the technical division at ILTS.

*To whom correspondence should be addressed. watanabe@lowtem.hokudai.ac.jp

${ }^{\dagger}$ Present address: National Institute for Materials Science, Tsukuba, Ibaraki 305-0047 Japan.

[1] L. Pagani, E. Roueff, and P. Lesaffre, Astrophys. J. 739, L35 (2011).

[2] K. Fukutani and T. Sugimoto, Prog. Surf. Sci. 88, 279 (2013).

[3] E. Ilisca, Prog. Surf. Sci. 41, 217 (1992).

[4] N. Wakao, J. M. Smith, and P. W. Selwood, J. Catal. 1, 62 (1962).

[5] D. Ashmead, D. D. Eley, and R. Rudham, J. Catal. 3, 280 (1964).

[6] K. Fukutani, K. Yoshida, M. Wilde, W. A. Diño, M. Matsumoto, and T. Okano, Phys. Rev. Lett. 90, 096103 (2003).

[7] K. Niki, T. Kawauchi, M. Matsumoto, K. Fukutani, and T. Okano, Phys. Rev. B 77, 201404 (2008).

[8] P. Avouris, D. Schmeisser, and J. E. Demuth, Phys. Rev. Lett. 48, 199 (1982).

[9] E. Ilisca, Phys. Rev. Lett. 66, 667 (1991).

[10] S. Andersson and J. Harris, Phys. Rev. Lett. 48, 545 (1982).

[11] K. Svensson and S. Andersson, Phys. Rev. Lett. 98, 096105 (2007).

[12] R. E. Palmer and R. F. Willis, Surf. Sci. 179, L1 (1987).

[13] S. Yucel, N. Alexander, and A. Honig, Phys. Rev. B 42, 820 (1990).

[14] S. A. FitzGerald, J. Hopkins, B. Burkholder, M. Friedman, and J. L. C. Rowsell, Phys. Rev. B 81, 104305 (2010).

[15] M. Chehrouri, J.-H. Fillion, H. Chaabouni, H. Mokrane, E. Congiu, F. Dulieu, E. Matar, X. Michaut, and J. L. Lemaire, Phys. Chem. Chem. Phys. 13, 2172 (2011).
[16] T. Hama, K. Kuwahata, N. Watanabe, A. Kouchi, Y. Kimura, T. Chigai, and V. Pirronello, Astrophys. J. 757, 185 (2012).

[17] T. Sugimoto and K. Fukutani, Nat. Phys. 7, 307 (2011).

[18] N. Watanabe, Y. Kimura, A. Kouchi, T. Chigai, T. Hama, and V. Pirronello, Astrophys. J. Lett. 714, L233 (2010).

[19] as a review, see N. Watanabe and A. Kouchi, Prog. Surf. Sci. 83, 439 (2008).

[20] C. Girardet and C. Toubin, Surf. Sci. Rep. 44, 159 (2001).

[21] L. Amiaud, J. H. Fillion, S. Baouche, F. Dulieu, A. Momeni, and J. L. Lemaire, J. Chem. Phys. 124, 094702 (2006).

[22] H. Katsuki and T. Momose, Phys. Rev. Lett. 84, 3286 (2000).

[23] Y. Miyamoto, M. Fushitani, D. Ando, and T. Momose, J. Chem. Phys. 128, 114502 (2008).

[24] P. L. Scott and C. D. Jeffries, Phys. Rev. 127, 32 (1962).

[25] T. Sugimoto, K. Yamakawa, and I. Arakawa, J. Chem. Phys. 143, 224305 (2015).

[26] H. Hidaka, N. Miyauchi, A. Kouchi, and N. Watanabe, Chem. Phys. Lett. 456, 36 (2008).

[27] P. Ayotte, R. S. Smith, K. P. Stevenson, Z. Dohnálek, G. A. Kimmel, and B. D. Kay, J. Geophys. Res. Planets 106, 33387 (2001).

[28] See Supplemental Material at http://link.aps.org/ supplemental/10.1103/PhysRevLett.116.253201 for experimental procedure detail; the conversion for the $\mathrm{D}_{2}$ molecule; and the conversion rate for $\mathrm{H}_{2}$ at $10 \mathrm{~K}$ when $\mathrm{H}_{2}$ was initially deposited at $12 \mathrm{~K}$ and immediately cooled to $10 \mathrm{~K}$.

[29] T. Kosone, A. Hori, E. Nishibori, Y. Kubota, A. Mishima, M. Ohba, H. Tanaka, K. Kato, J. Kim, J. A. Real, S. Kitagawa, and M. Takata, R. Soc. Open Sci. 2, 150006 (2015).

[30] L. Hornekaer, A. Baurichter, V. V Petrunin, A. C. Luntz, B. D. Kay, and A. Al-Halabi, J. Chem. Phys. 122, 124701 (2005).

[31] A. J. Leadbetter, Proc. R. Soc. London A Math. Phys. Eng. Sci. 287, 403 (1965).

[32] O. Yamamuro, Y. Madokoro, H. Yamasaki, T. Matsuo, I. Tsukushi, and K. Takeda, J. Chem. Phys. 115, 9808 (2001).

[33] A. Glebov, A. P. Graham, A. Menzel, J. P. Toennies, and P. Senet, J. Chem. Phys. 112, 11011 (2000).

[34] J. Van Kranendonk, Physica (Amsterdam) 20, 781 (1954).

[35] E. Ilisca and F. Ghiglieno, Eur. Phys. J. B 87, 235 (2014).

[36] G.-J. Kroes and C. Diaz, Chem. Soc. Rev. (2016).

[37] D. Farías and R. Miranda, Prog. Surf. Sci. 86, 222 (2011). 